Pacific Journal of Mathematics

INVARIANT MEANS AND ERGODIC SETS IN FOURIER 


\section{INVARIANT MEANS AND ERGODIC SETS IN FOURIER ANALYSIS}

\section{GORDON S. WOODWARD}

Let $\phi$ be a uniformly continuous function on the locally compact, non-compact, abelian group $G$. Denote the dual of $G$ by $\Gamma$. Then $\phi$ is ergodic on $E \subset \Gamma$ if $\phi \gamma$ has a unique (translation-invariant) mean value for et ch $\gamma \in E$. Theorem: If $E$ is closed and scattered, then there is a sequence $\left\{p_{n}\right\}$ of trigonometric polynomials and a sequence $\left\{g_{n}\right\} \subset L^{1}(G)$ with supp $\hat{g}_{n} \cap E=\phi$ such that $g_{n}{ }^{*} \phi+p_{n}$ $\rightarrow \phi$ uniformly. Now suppose $E=\Gamma \backslash\{\gamma\}$ and let $\left\{p_{\beta}\right\}$ be any net of almost periodic functions on $G$ with bounded $L^{1}(\bar{G})$ norm. Theorem: All cluster points to $\left.\left.\left\{M_{y}\right) p_{\beta}(\cdot-y) \phi(y)\right)\right\}_{\beta}$ in the topology of uniform convergence on compact subsets of $G$ are ergodic. Here $M$ is any invariant mean on $G$. A compact set $E \subset \Gamma$ is ergodic if each $\phi \in \Phi(E)$, the $L^{\infty}(G)$-weak* closure of span $E$, is (equal a.e. to) a function ergodic on $\Gamma$.Theorem: If $E$ is ergodic and $S$ is compact scattered, then $E \cup S$ is ergodic. For $E$ 's which are Helson sets this implies that the elements of $\Phi(E \cup S)$ decompose into $\hat{\mu}+u$ where $\mu \in M_{c}(E)$ and $u$ is almost periodic.

Let $\operatorname{IM}(G)$ be the set of all positive, translation-invariant, linear forms on the space $C(G)$ of uniformly continuous, complex-valued, bounded functions on $G$. We say a function $\phi \in C(G)$ is ergodic on $E \subset \Gamma$ if the mean value $M(\phi \bar{\gamma})$ is independent of $M \in I M(G)$ for each $\gamma \in E$; if $E=\Gamma, \phi$ is simply called ergodic. A compact set $E \subset \Gamma$ is ergodic if each $\phi \in \Phi(E)$ is ergodic, where $\Phi(E)$ is the weak* closure of the span of $E$ in $L^{\infty}(G)$ (each $\phi \in \Phi(E)$ is equal a.e. to an element of $C(G)$ since $E$ is compact).

The class $\mathscr{E}(G)$ of ergodic functions on $G$ properly contains the weakly almost periodic (WAP) functions which were introduced by Eberlein in [3], and it is equivalent to the class of Ryll-Nardzewski functions in $C(G)$ for $G=R$, as studied by Kahane in [6]. Our work here is heavily influenced by Eberlein's study. Because of this we consider ergodic functions as generalized WAP functions. But the analogy is distant. For in [6] it is proved that any $\phi \in C(R)$ is the product of two ergodic functions! In spite of this, ergodic functions share some strong approximation properties with WAP functions. For example our Theorems 8 and 9 (ii) yield:

TheOREM. Suppose $\phi$ is ergodic on the closed scattered set $S$. Then there exist trigonometric polynomials $p_{n}$ with spectra in $S$ and $L^{1}(G)$ functions $g_{n}$ with closed spectra disjoint from $S$ such that $g_{n}{ }^{*} \phi+p_{n} \rightarrow \phi$ uniformly on $G$. 
THEOREM. Fix $\gamma \in \Gamma$, let $\epsilon>0$, and suppose $\phi$ is ergodic on $\Gamma \backslash\{\gamma\}$. Then there is a neighborhood $U$ of $\gamma$ such that for any $f \in L^{1}(G)$ with supp $\hat{f}$ $\subset U$ and for any trigonometric polynomial $\sum_{\Gamma} a_{\eta} \eta$ with $a_{\gamma}=0$ one has

$$
\left\|f^{*}\left(\sum_{\Gamma} a_{\eta} M(\phi \bar{\eta}) \eta\right)\right\|_{\infty} \leq \epsilon\left\|\sum_{\Gamma} a_{\eta} M(\phi \bar{\eta}) \eta\right\|_{\infty}\|f\|_{1}
$$

for any $M \in I M(G)$.

These theorems indicate the wholly ergodic nature of the result of Loomis in [9] and are actually extensions of the key lemmas in that article. They might also suggest two questions which we comment on now. First, it is not difficult to construct functions ergodic on $\Gamma \backslash\{\gamma\}$ and not ergodic on $\{\gamma\}$. Thus the hypothesis in the latter theorem is not superfluous. Second, there are ergodic $\phi$ 's for which the function $\mathscr{F}(\phi)(\gamma) \equiv M(\phi \bar{\gamma})$ is not the Bohr-Fourier transform of any uniformly almost periodic or even any Weyl-almost periodic function on $R$. For a discussion of this see [14] and compare with [4].

Ergodic sets were introduced in [13] as a class of thin sets in the Fourier analytic sense. There it is shown that $\Phi(E) \cap C_{0}(G)=\{0\}$ is necessary for $E$ to be ergodic (i.e., ergodic sets are weak uniqueness sets) and that ergodic sets cannot contain perfect symmetric sets. Compact scattered sets are ergodic by the Loomis result [9] and, of course, Helson sets are ergodic. However there do exist perfect ergodic sets which contain arbitrarily large arithmetic progressions. Such an example is constructed by Katznelson and McGehee in [7]. Otherwise little is known about this class. For example: Is the union of two ergodic sets again ergodic? What is the relationship between ergodic sets and the classical thin sets? Also, we need more necessary properties of ergodic sets. Here we prove the seemingly elementary result that the union of an ergodic set with a compact scattered set is again ergodic (Theorem 12). Its proof depends heavily on the two theorems mentioned previously, and it has the following corollary: if $E$ is Helson and $S$ is compact scattered, then each $\phi \in \Phi(E \cup S)$ decomposes into $\phi=\hat{\mu}+u$ where $\mu \in M(E)$ and $u$ is uniformly almost periodic.

Fundamental to our work is the characterization of the mean values of any $\phi \in C(G)$ in terms of a class of convolution kernels which we call averaging kernels. In general these kernels give rise to most of the classical averaging techniques including those found in [1]. But the averaging kernels we find most useful are nets consisting of elementary positive definite functions whose spectra form a neighborhood base for $0 \in \Gamma$. These kernels are discussed in $\S 2$ while $\S \S 3$ and 4 deal with ergodic functions and 
ergodic sets, respectively. The first section is reserved for preliminaries and notation.

We wish to thank Professor C. Robert Warner for many stimulating discussions concerning this material.

Preliminaries. By the translate of a function $\phi$ on $G$, we mean the function $\phi_{x}(y) \equiv \phi(y-x)$ for some $x \in G$. The set of all translates of $\phi$ is $\operatorname{Tr}(\phi)$, and $R(\phi)$ is the range of $\phi$. Denote the convex hull, interior, complement and closure of a set $A$ by $\operatorname{co}(A), A^{\circ}, A^{c}$, and $\bar{A}$, respectively.

The space $C(G)$ is endowed with the supremum norm, $\|\cdot\|_{\infty}$. The operations and partial ordering are defined pointwise in the usual manner. A trigonometric polynomial on $G$ is any finite complex linear combination of elements of $\Gamma$. The set of all such polynomials is denoted by $P(G)$. Its closure in $C(G)$ is $A P(G)$, the algebra of (uniformly) almost periodic functions.

The space of finite, regular, Borel measures on $G$ is denoted by $M(G)$. Its norm, $\|\cdot\|$, is the total variation norm. $L^{1}(G)$ and $L^{\infty}(G)$ are the usual spaces of Haar-measurable functions. Fix a Haar measure on $G$ and its dual Haar measure on $\Gamma$. Unless otherwise stated all integrals are taken with respect to these fixed measures. The Haar measure of a set $A$ is $|A|$. The support of a function or measure $u$ is denoted by supp $u$. Recall that $M(G)$ and $L^{1}(G)$ are Banach algebras under the convolution product *. We denote the Fourier (-Stieltjes) transform of a $u \in L^{1}(G)(M(G)$ ) by $\hat{u}$.

The spectrum of a $\phi \in L^{\infty}(G)$ is the intersection of $\Gamma$ with the weak* closure of the span of $\operatorname{Tr}(\phi)$. It is denoted by $\sigma(\phi)$. Equivalently, $\sigma(\phi)$ is the zero set of the ideal $I$ consisting of all $f \in L^{1}(G)$ such that $f^{*} \phi \equiv 0$. That is

$$
\sigma(\phi)=\{\gamma \in \Gamma \mid \hat{f}(\gamma)=0 \text { for all } f \in I\}
$$

For $f \in L^{1}(G)$ its closed spectrum is supp $\hat{f}$. Given a subset $E \subset \Gamma$, we define $\Phi(E)$ to be the weak* closure of the span of $E$ in $L^{\infty}(G)$. If $E$ is precompact, then each $\phi \in \Phi(E)$ is equal a.e. to an element of $C(G)$, so we assume $\phi \in C(G)$. In particular for precompact $E$ 's, $\Phi(E)$ is the closure of span $E$ in the relative $L^{\infty}(G)$-weak* topology on $C(G)$ (see [5, Preliminaries]). We say $E \subset \Gamma$ is a spectral set if $\sigma(\phi) \subset E$ implies $\phi \in \Phi(E)$. Finite sets are spectral sets; hence if $E$ is finite, $\Phi(E) \equiv\left\{\sum_{E} a_{\gamma} \gamma: a_{\gamma} \in \mathbf{C}\right\}$.

An (translation-) invariant mean $M$ on $C(G)$ is a positive linear form on $C(G)$ satisfying (i) $M(1)=1$ and (ii) $M(\psi)=M(\phi)$ for all $\psi \in \operatorname{Tr}(\phi)$. We denote by $I M(G)$ the set of all invariant means on $G$. Note that $M \in I M(G)$ implies $M$ is continuous with operator norm $\|M\|=1$. The number $M(\phi)$ is 
called a mean value of $\phi$. We denote the set of all mean values of $\phi$ by $M V(\phi)$.

There are two basic facts concerning invariant means which we list now. Because of their importance, we also give an outline of their proofs. The first will hereafter be referred to as the Fundamental Characterization Theorem. It can be found in [5, Theorem 2.1].

(1) Suppose $\phi \in C(G)$. Then

$$
M V(\phi)=\cap\{\overline{\operatorname{co}}(R(\psi)) \mid \psi \in \operatorname{co}(\operatorname{Tr}(\phi))
$$

(2) Suppose $M \in I M(G), \phi \in C(G)$, and $u \in L^{1}(G)$ (or $M(G)$ ). Then

$$
M\left(u^{*} \phi\right)=\hat{u}(0) M(\phi)
$$

To begin the proof of (1), we show LHS ᄃ RHS. Suppose $c \notin \overline{\operatorname{co}}(\operatorname{Tr}(\psi))$ for some $\psi \in \operatorname{Tr}(\phi)$. Since a compact convex set is the intersection of all half spaces ontaining it, it is also the intersection of all discs containing it. Thus there is a disc $D$ with center $b$ such that $c \notin D$ and $R(\psi) \subset D$. Therefore $|M(\phi)-b| \leq M(|\psi-b|) \leq\|\psi-b\|_{\infty}<|c-b|$. Conversely, suppose $c \in R H S$ of (1). Then define the linear map $L$ on the linear space $\{a+b \phi \mid a, b \in \mathbf{C}\}$ by $L(a+b \phi) \equiv a+b c$. This map is dominated by the function $T(\phi) \equiv \inf \|\mathrm{X}\|_{\infty}$ over all $\mathrm{X} \in \operatorname{co}(\operatorname{Tr}(\phi))$. Moreover one can verify that $T$ is sublinear $(T(\psi+\phi) \leq T(\psi)+T(\phi)$ and $T(\alpha \psi)=|\alpha| T(\psi))$ and that $T\left(\psi-\psi_{x}\right)=0$ for any $x \in G$ on $C(G)$. In particular, $L$ extends via the Hahn-Banach Theorem to a continuous linear functional $M$ on $C(G)$ which is dominated by $T$; hence $M \in I M(G)$ and $M(\phi)=c$. Statement (2) is a consequence of the elementary fact that $u^{*} \phi$ is the uniform limit of functions of the form

$$
\sum_{i=1}^{\infty} \int_{V_{i}} u(x) d x \phi_{x_{i}}
$$

where $\left\{V_{i}\right\}$ is a measurable partition of supp $u$ and $x_{i} \in V_{i}$, whenever $\phi \in$ $C(G)$.

As general references we suggest [11], for the Fourier analysis; [5], for invariant means; and [1] or [2], for almost periodic functions. Our notation is taken from [11] whenever possible

Averaging kernels. We will need a systematic method for describing the mean values of a function. The literature abounds with general summability methods. But here the method must be useful, simple, and particularly suitable for the investigation of the local spectrum of a function. 
The one we will use is essentially a "system of almost invariant integrals" as defined by Eberlein in [3, Definition 2.1]. It is closely related to the summability method of Lorentz in [10, Theorem 7].

Definition 1. An averaging kernel on $G$ is a net $\left\{k_{\alpha}\right\}$ in $L^{1}(G)$ which satisfies

(i) $\lim \left\|k_{\alpha}\right\|_{1}=\lim \hat{k}_{\alpha}(0)=1$ and

(ii) $\lim _{\alpha}\left\|k_{\alpha}-\left(k_{\alpha}\right)_{x}\right\|_{1}=0$ for all $x \in G$.

The following construction gives rise to an important class of averaging kernels. Let $\left\{U_{\alpha}\right\}$ be a neighborhood base for $0 \in \Gamma$ consisting of compact symmetric sets which is partially ordered by $\alpha \leq \beta$ if and only if $U_{\alpha} \supset U_{\beta}$. Denote by $\mathrm{X}_{\alpha}$ the characteristic function of $U_{\alpha}$. Now, using [11, Theorem 1.6.3], set

$$
\hat{f}_{\alpha}=\left|U_{\alpha}\right|^{-1} \mathrm{X}_{\alpha} * \mathrm{X}_{\alpha}
$$

Since $\hat{f}_{\alpha}$ is positive definite with $\hat{f}_{\alpha}(0)=1$, we conclude that $\left\|f_{\alpha}\right\|_{1}=1$. That $\lim \left\|f_{\alpha}-\left(f_{\alpha}\right)_{x}\right\|_{1}=0$ for each $x \in G$ follows from the fact that points are spectral sets. Indeed, fix $\epsilon>0$ and $x \in G$ and choose $g \in L^{\prime}(G)$ so that $\hat{g} \equiv$ 1 on a neighborhood of $0 \in \Gamma$. Then there is an $h \in L^{1}(G)$ with $\hat{h} \equiv 0$ on some neighborhood of $0 \in \Gamma$ such that $\left\|g-g_{x}-h\right\|_{1}<\epsilon$. Thus for $\alpha$ sufficiently large

$$
\begin{gathered}
\left\|f_{\alpha}-\left(f_{\alpha}\right)_{x}\right\|_{1}=\left\|\left(g-g_{x}\right)^{*} f_{\alpha}\right\|_{1} \\
\leq\left\|g-g_{x}-h\right\|_{1}\left\|f_{\alpha}\right\|_{1}<\epsilon .
\end{gathered}
$$

Therefore $\left\{f_{\alpha}\right\}$ is an averaging kernel on $G$. Observe that if $\left\{U_{\alpha}\right\}$ were partially ordered by $\alpha \leq \beta$ if and only if $U_{\alpha} \subset U_{\beta}$ and if $G=U_{\alpha}>\beta U_{\alpha}$ for every choice of $\beta$, then the same construction would lead to a Fejér kernel. Because of this we call $\left\{f_{\alpha}\right\}$ a Fejér averaging kernel on $G$. More generally, $\left\{k_{\alpha}\right\}$ is a Fejér averaging kernel if $\left\{k_{\alpha}\right\}$ is an averaging kernel which satisfies (1) $\left\|k_{\alpha}\right\|_{1}=\hat{k}_{\alpha}(0)=1$ and (2) $\left\{\operatorname{supp} \hat{k}_{\alpha}\right\}$ is a neighborhood base for $0 \in \Gamma$. Evidently $\left\{k_{\alpha}\right\}$ must consist of non-negative functions.

Let $D_{r}$ denote the closed disc of radius $r$ with center at the origin in the complex plane. We then describe the relationship between mean values and averaging kernels as follows: 
TheOREM 2. Let $\phi \in C(G)$ and suppose that $\left\{k_{\alpha}\right\}$ is an averaging kernel on $G$. Then for each $\epsilon>0$ there is an $\alpha_{\epsilon}$ such that

$$
M V(\phi) \subset \overline{\operatorname{co}}\left(R\left(k_{\alpha}^{*} \phi\right)\right)+D_{\epsilon} \subset M V(\phi)+D_{2 \epsilon}, \text { for } \alpha \geq \alpha_{\epsilon} .
$$

Furthermore if $\left\{k_{\alpha}\right\}$ is a Fejér averaging kernel, then

$$
M V(\phi) \subset \overline{\operatorname{co}}\left(R\left(k_{\alpha}^{*} \phi\right)\right) \text { for all } \alpha
$$

Proof. The second statement is a consequence of the Fundamental Characterization Theorem and the fact that $g^{*} \phi \in \overline{\operatorname{co}}(\operatorname{Tr}(\phi))$ whenever $g$ $\in L^{1}(G)$ satisfies $\|g\|_{1}=\hat{g}(0)=1$.

To prove the first statement, we begin with a Fejér averaging kernel $\left\{k_{\alpha}\right\}$. In this case the first inclusion has just been verified and one need only prove the second. Clearly, it is sufficient to show that $\operatorname{co}\left(R\left(k_{\alpha} * \phi\right) \subset\right.$ $M V(\phi)+D_{\epsilon}$ for all $\alpha$ sufficiently large (depending on $\epsilon>0$ ). To this end, the Fundamental Characterization Theorem together with a compactness argument implies the existence of $\psi_{1}, \ldots, \psi_{n} \in \operatorname{co}(\operatorname{Tr}(\phi))$ such that $\cap_{j} \operatorname{co}\left(R\left(\psi_{j}\right)\right) \subset M V(\phi)+D_{r}$, where $r=\epsilon / 2$. Since

$$
\lim _{\alpha}\left\|k_{\alpha} * \phi-k_{\alpha}^{*} \psi\right\|_{\infty}=0
$$

for any $\psi \in \operatorname{co}(\operatorname{Tr}(\phi))$ by Definition 1 (ii), it follows that $\operatorname{co}\left(R\left(k_{\alpha}{ }^{*} \phi\right)\right) \subset$ $M V(\phi)+D_{r}+D_{r}=M V(\phi)+D_{\epsilon}$ for all $\alpha$ sufficiently large. For the general averaging kernel $\left\{k_{\alpha}\right\}$ it is enough to note that Definition 1 (i) implies that the imaginary and negative real parts of $k_{\alpha}$ limit to zero in $L^{1}$-norm as $\alpha \rightarrow \infty$.

Theorem 2 has an important corollary which several authors have observed in various forms (see [3, Theorem 3.1], [8, §6] and [10, Theorem 1]). We state it as follows:

Corollary 3. Let $\left\{k_{\alpha}\right\}$ be an averaging kernel on G. Suppose $\phi \in$ $C(G)$ and fix $\gamma \in \Gamma$. The following statements are equivalent.

(i) $\phi \bar{\gamma}$ has a unique mean value.

(ii) $\left\{k_{\alpha}^{*}(\phi \bar{\gamma})\right\}$ is uniformly Cauchy.

(iii) $\left\{\left(k_{\alpha} \gamma\right)^{*} \phi\right\}$ converges uniformly to $M(\phi \bar{\gamma}) \gamma$, for any invariant mean $M$ on $C(G)$.

Proof. According to Theorem 2, (i) is equivalent to (ii); (ii) implies 
(iii) by this equivalence and the identity $\left(k_{\alpha} \gamma\right)^{*} \phi \equiv\left[k_{\alpha} *(\phi \bar{\gamma})\right] \gamma$. Now (iii) implies (i) is immediate.

Ergodic functions. In [3] Eberlein uses his system of almost invariant integrals to investigate "ergodic points" in certain Banach spaces. We will use averaging kernels to investigate "ergodic functions" in $C(G)$. However, our definition of ergodic function is considerably more restrictive than that of ergodic point. Indeed an ergodic point in $C(G)$ becomes in our terminology a function ergodic at zero. Since we are primarily interested in functions which are ergodic on infinite sets, it is not surprising that the analogy between our methods and those of Eberlein stop at Corollary 3.

Definition 4. A $\phi \in C(G)$ is ergodic at $\gamma \in \Gamma$ if $\phi \bar{\gamma}$ has a unique mean value. The function $\phi$ is ergodic on $E \subset \Gamma$ if $\phi$ is ergodic at each $\gamma \in$ $E$; if $E=\Gamma$, then $\phi$ is called ergodic.

It is immediate that the class of functions ergodic at a fixed $\gamma \in \Gamma$ is a uniformly closed translation-invariant subspace of $C(G)$; hence so must be $\mathscr{E}(G)$, the class of ergodic functions. Moreover $\mathscr{E}(G)$ is closed under complex conjugation and under composition with the map $x \rightarrow-x$. Observe also that $\mathscr{E}(G)$ contains the algebra of Weyl almost periodic functions in $C(G)$ (see [1]) and, in particular, the algebras of $W A P$ and $A P$ functions on $G$. For more comments on $\mathscr{E}(G)$ see the introduction.

We also remark that our concept of ergodicity can be extended to functions in $L^{\infty}(G)$. When this is done, Theorem 2 is valid with some modifications.

In this section we obtain the two approximation results mentioned in the introduction. The first, Theorem 8 , is concerned with the "local" approximation of an ergodic function by almost periodic functions. Precisely what this means is defined next. The more technical aspects of its proof are given in Lemmas 6 and 7. The second, Theorem 9, we comment on later.

Definition 5. Let $E \subset \Gamma$. A $\phi \in C(G)$ is E-approximable if there exist sequences $\left\{p_{n}\right\} \subset P(G)$ and $\left\{g_{n}\right\} \subset L^{1}(G)$ such that

(i) $\sigma\left(p_{n}\right) \subset E$,

(ii) $\operatorname{supp} \hat{g}_{n} \cap E=\phi$, and

(iii) $\left\{g_{n} * \phi+p_{n}\right\}$ converges uniformly to $\phi$.

Any pair $\left\{g_{n}\right\},\left\{p_{n}\right\}$ which satisfies these conditions will be called a ( $\phi$, E)-pair.

The set of $E$-approximable functions is evidently a translation-invariant subset of $C(G)$. Moreover it is closed under convolution with $L^{1}(G)$ 
and, in fact, with $M(G)$. Furthermore since invariant means are continuous functionals on $C(G)$ and since $P(G) \subset \mathscr{E}(G)$, every $E$-approximable function $\phi$ is ergodic on $E$; if $E^{\circ} \neq \phi$ then $\phi$ must be locally almost periodic on $E^{\circ}$. In this sense, $E$ approximation extends the concept of local almost periodicity as defined in [9].

Lemma 6. Fix $\gamma \in \Gamma$ and suppose $\phi$ is ergodic at $\gamma$. Then $\phi$ is $\{\gamma\}$-approximable.

Proof. Let $\left\{k_{\alpha}\right\}$ be a Fejér averaging kernel on $G$ and choose a bounded approximate identity $\left\{h_{\beta}\right\}$ for $L^{1}(G)$ which satisfies $\hat{h}_{\beta}(0)=1$ for all $\beta$. Then for each positive integer $n$ there is a $\beta_{n}$ such that

$$
\left\|\phi-\left(h_{\beta_{n}} \gamma\right) * \phi\right\|_{\infty}<n^{-1}
$$

and, by Corollary 3 (iii), an $\alpha_{n}$ such that

$$
\left\|\left(k_{\alpha_{n}} \gamma\right)^{*} \phi-M(\phi \bar{\gamma}) \gamma\right\|_{\infty}<n^{-1}
$$

Here $M$ is any invariant mean. Since points are spectral sets and since $\left(h_{\beta_{n}} \gamma\right.$ $\left.-k_{\alpha_{n}} \gamma\right)^{\wedge}(\gamma)=0$, there exists a $g_{n} \in L^{1}(G)$ with $\hat{g}_{n}=0$ on a neighborhood of $\gamma$ such that

$$
\left\|\left(h_{\beta_{n}} \gamma\right)-\left(k_{\alpha_{n}} \gamma\right)-g_{n}\right\|_{1}<n^{-1}\left(\|\phi\|_{\infty}+1\right)^{-1}
$$

We conclude

$$
\begin{aligned}
& \left\|g_{n}{ }^{*} \varphi+M(\varphi \gamma) \gamma-\varphi\right\|_{\infty} \leq \\
& \left\|\varphi-\left(h_{\beta_{n}} \gamma\right)^{*} \varphi\right\|_{\infty}+\left\|\left(h_{\beta_{n}} \gamma-k_{\alpha_{n}} \gamma\right)^{*} \varphi-g_{n}{ }^{*} \varphi\right\|_{\infty}+ \\
& \left\|\left(k_{\alpha_{n}} \gamma\right)^{*} \varphi-M(\varphi \gamma) \gamma\right\|_{\infty}<3 n^{-1} .
\end{aligned}
$$

It is now easy to verify that $\left\{g_{n}\right\},\{M(\phi \bar{\gamma}) \gamma\}$ is a $(\phi,\{\gamma\})$-pair.

LEMMA 7. Let $E \subset \Gamma$ be compact and fix $\xi \in E$. Assume that $\phi \in C(G)$ is ergodic on $E$. If for each $\gamma \in E$ except $\xi$ there is a neighborhood $V$ of $\gamma$ such that $\phi$ is $V \cap E$-approximable, then $\phi$ is E-approximable.

Proof. We will exhibit an arbitrary element of a $(\phi, E)$-pair. Fix a positive integer $n$. According to Lemma 6, there exist functions $f_{n}, p_{n}$ which 
belong to a $(\phi,\{\xi\})$-pair such that $\left\|f_{n}{ }^{*} \phi+p_{n}-\phi\right\|_{\infty}<n^{-1}$. Let $V_{1}$ be a compact neighborhood of $\xi$ which satisfies $V_{1} \cap \operatorname{supp} \hat{f}_{n}=\phi$. If our hypothesis is valid the compactness of $E$ implies that there is a collection $V_{2}, \ldots, V_{m}$ of compact subsets of $\Gamma$ satisfying

$$
E \subset \bigcup_{i=1}^{m} V_{i}^{\circ} \quad \text { and }
$$

$\phi$ is $V_{j} \cap E$-approximable for $2 \leq j \leq m$.

Using a standard argument [11, proof of Lemma 6.26], one can choose the sets $V_{1}, \ldots, V_{m}$ so that there exist functions $g_{1}, \ldots, g_{m} \in L^{1}(G)$ which satisfy
(1) $\hat{\mathrm{g}}_{i}>0$ for $1 \leq i \leq m$,
(2) $\hat{g}_{i} \equiv 0$ on $V_{i}^{c}$ for $1 \leq i \leq m$,
(3) $\sum_{i=1}^{m} \hat{g}_{i} \equiv 1$ on a neighborhood of $E$.

(One can even show that $\left\|g_{1}\right\|_{1} \leq 2\left[11\right.$, Theorem 2.6.3].) Now let $\left\{f_{k}^{(i)}\right]$, $\left\{p_{k}^{(i)}\right\}$ be a $\left(\phi, V_{i} \cap E\right)$-pair for $2 \leq i \leq m$, which exists by hypothesis. It then follows that $\left\{f_{k}^{(i)}\right\},\left\{g_{i}{ }^{*} p_{k}^{(i)}\right\}$ is a $\left(g_{i}{ }^{*} \phi, V_{i} \cap E\right)$-pair. In particular, $\left\{f_{k}^{(i) *} g_{i}{ }^{*} \phi+g_{i}{ }^{*} p_{k}{ }^{(i)}\right\}$ converges uniformly to $g_{i} * \phi$. Therefore there is a subscript $k_{n}$, which we call $n$ for simplicity, such that

(4) $\left\|g_{1}{ }^{*} f_{n}{ }^{*} \varphi+\sum_{i=2}^{m} g_{i}{ }^{*} f_{n}{ }^{(i) *} \varphi+g_{1}{ }^{*} p_{n}+\sum_{i=2}^{m} g_{i}{ }^{*} p_{n}{ }^{(i)}-\sum_{i=1}^{m} g_{i}{ }^{*} \varphi\right\|_{\infty}<3 n^{-1}$.

Finally, choose $h_{n} \in L^{1}(G)$ so that $\hat{h}_{n} \equiv 1$ on a neighborhood of $E$ and so that $\left\|h_{n}^{*} \phi-\phi\right\|_{\infty}<n^{-1}$. Set

$$
\begin{gathered}
k_{n}=g_{1}{ }^{*} f_{n}+\sum_{i=2}^{m} g_{i}{ }^{*} f_{n}^{(i)}+h_{n}-\sum_{i=1}^{m} g_{i} \quad \text { and } \\
q_{n}=g_{1}{ }^{*} p_{n}+\sum_{i=2}^{m} g_{i}{ }^{*} p_{n}{ }^{(i)}
\end{gathered}
$$

We claim that $k_{n}, q_{n}$ represent a general element of a $(\phi, E)$-pair. To this end, (2) and (3) together with the properties of $f_{n}, f_{n}^{(i)}$ and $h_{n}$ yield 


$$
\begin{gathered}
\left(\operatorname{supp} \hat{k}_{n} \cap E\right) \subset\left[\left(\operatorname{supp} \hat{g}_{1} \cap \operatorname{supp} \hat{f}_{n}\right) \bigcup_{i=2}^{m}\left(\operatorname{supp} \hat{g}_{i} \cap \operatorname{supp} \hat{f}_{n}^{(i)}\right)\right. \\
\left.\cup \operatorname{supp}\left(h_{n}-\sum_{i=1}^{m} g_{i}\right)^{\wedge}\right] \cap E \\
\subset\left[\left(V_{1} \cap V_{1}^{c}\right) \bigcup_{i=2}^{m}\left(V_{i} \cap\left(V_{i} \cap E\right)^{c}\right) \cup E^{c}\right] \cap E=\varnothing,
\end{gathered}
$$

while (3) and (4) imply

$$
\begin{aligned}
\left\|k_{n}{ }^{*} \varphi+q_{n}-\varphi\right\|_{\infty} & \leq\left\|\left[k_{n}-\left(h_{n}-\sum_{i=1}^{m} g_{i}\right)\right]^{*} \varphi+q_{n}-\sum_{i=1}^{m} g_{i}{ }^{*} \varphi\right\|_{\infty} \\
& +\left\|\left(h_{n}-\sum_{i=1}^{m} g_{i}\right)^{*} \varphi+\sum_{i=1}^{m} g_{i}^{*} \varphi-\varphi\right\|_{\infty} \\
& <3 n^{-1}+n^{-1}=4 n^{-1} .
\end{aligned}
$$

The connection between ergodicity and $E$-approximation may now be described.

THEOREM 8. Suppose $\phi \in C(G)$ is ergodic on the closed scattered set $S$. Then $\phi$ is $S$-approximable. Define

Proof. Assume for now that $\sigma(\phi)$ is compact and set $E=\sigma(\phi) \cap S$.

$$
\begin{gathered}
Q=\{\gamma \in E \mid \phi \text { is not } V \cap E \text {-approximable } \\
\text { for any neighborhood } V \text { of } \gamma\} .
\end{gathered}
$$

We claim that $Q=\varnothing$. For otherwise $Q$, being a subset of $E$, contains an isolated point $\xi$. Thus, there is a neighborhood $V$ of $\xi$ such that $V \cap Q=$ $\{\xi\}$. Choose $g \in L^{1}(G)$ so that $\hat{g} \equiv 1$ on a neighborhood of $\xi$ and so that supp $\hat{g} \subset V$. Set $k=h-g$, where $h \in L^{1}(G)$ with $\hat{h} \equiv 1$ on a neighborhood of $\sigma(\phi)$. Since $\xi \notin \sigma\left(k^{*} \phi\right)$, there is some neighborhood $U$ of $\xi$ such that $k^{*} \phi$ is $U \cap E$-approximable. Furthermore, $g^{*} \phi$ satisfies the hypothesis of Lemma 7 by our choice of $g$ and $\xi$. Thus $\phi \equiv k^{*} \phi+g^{*} \phi$ is $U$ $\cap E$-approximable for some neighborhood $U$ of $\xi$. We conclude that $\xi \notin$ $Q$, which is a contradiction.

Since $Q=\phi$, Lemma 7 implies that $\phi$ is $E$-approximable. It remains to show that $\phi$ is $S$-approximable. Let $\left\{g_{n}\right\},\left\{p_{n}\right\}$ be a $(\phi, E)$-pair. Then 
(supp $\left.\hat{g}_{n} \cap \sigma(\phi)\right) \cap S=\phi$. Therefore there exist $f_{n} \in L^{1}(G)$ with supp $\hat{f}_{n} \cap$ $S=\phi$ such that $f_{n}{ }^{*} g_{n}{ }^{*} \phi=g_{n} * \phi$ for each $n$. Thus $\left\{f_{n}{ }^{*} g_{n}\right\},\left\{p_{n}\right\}$ is a $(\phi$, $S)$-pair.

Finally, the assumption that $\sigma(\phi)$ be compact can be removed by observing that any $\phi \in C(G)$ is the uniform limit of $\left\{k_{\alpha}{ }^{*} \phi\right\}$ where $\left\{k_{\alpha}\right\}$ is a bounded approximate identity for $L^{1}(G)$ with supp $\hat{k}_{\alpha}$ compact.

We remark that Theorem 8 is best possible in the following sense: if $S$ is a perfect closed set and if $\phi$ is the Fourier-Stieltjes transform of a nonzero continuous measure $\mu \in M(\Gamma)$ with support in $S$, then $\phi$ is ergodic on $S$ but not $S$-approximable. We also point out that under the hypothesis of Theorem 8 one can choose the $(\phi, S)$-pair $\left\{g_{n}\right\},\left\{p_{n}\right\}$ so that

$$
\sigma\left(p_{n}\right) \subset\{\gamma \in S \mid M(\phi \bar{\gamma}) \neq 0 \text { for } M \in I M(G)\} .
$$

This follows from the construction carried out in Lemma 7.

We now turn out attention from $E$-approximation to the behavior of trigonometric polynomials which are "naturally" related to a given $\phi \in$ $C(G)$ via an invariant mean. This will give rise to our second approximation result, Theorem 9. This theorem is somewhat reminiscent of the results in [9], and in fact is the key to our generalization of those results (see the comment following Theorem 12).

The nature of the remaining material gives rise to some technical difficulties if we restrict ourselves to elements in $C(G)$. To avoid this, we will consider functions on the Bohr compactification $\bar{G}$ of $G$ as well as functions on $G$. Working on both groups does, however, produce some notational problems which hopefully will be minimized by the following remarks.

Recall that $A P(G)$ is naturally, isometrically isomorphic to $C(\bar{G})$, so we will not distinguish between them. There is only one invariant mean on $A P(G)$; it is determined by integration on $\bar{G}$ with respect to its normalized Haar measure. In order to distinguish the action on $\bar{G}$ from $G$, we denote the convolution and $L^{p}$-norm on $\bar{G}$ by $\circ$ and $] I_{p}$, respectively. In both cases denotes transform. Thus for $p, q \in A P(G)$ and $M \in I M(G)$, we have $p \circ q(x)=M_{y}(p(x-y) g(y)),|p|_{1}=M(|p|)$, and $\hat{p}(\gamma)=M(p \bar{\gamma})$. There is a natural, norm preserving homomorphism $\rho: M(G) \rightarrow M(\bar{G})$ defined by

$$
\int_{\bar{G}} p d \rho(\mu) \equiv \int_{G} p d \mu \quad \text { for } \quad p \in A P(G) .
$$

Since each $f \in L^{1}(G)$ determines a unique measure in $M(G)$, the symbol $\rho(f)$ is a well defined element in $M(\bar{G})$. 
Fix $\phi \in C(G)$ and an invariant mean $M$ on $C(G)$ and suppose that $p=$ $\Sigma a_{i} \gamma_{i} \in P(G)$. Then we can define a trigonometric polynomial $q$ by $q(x)=$ $M_{y}(p(x-y) \phi(y))=\Sigma a_{i} M\left(\phi \bar{\gamma}_{i}\right) \gamma_{i}$. This polynomial is bounded by $M(|p|)\|\phi\|_{\infty}$. Also, $|q(x)-q(y)| \leq M(|p|)\left\|\phi_{-x}-\phi_{-y}\right\|_{\infty}$ for all $x, y \in G$. Thus if $\left\{p_{\beta}\right\} \subset P(G)$ is an $L^{1}(\bar{G})$ bounded approximate identity (for $\left.L^{1}(\bar{G})\right)$, then the net $\left\{M_{y}\left(p_{\beta}(\cdot-y) \phi(y)\right)\right\}$ is bounded and equicontinuous on $G$, and it is bounded on $\bar{G}$. In particular $\left\{M_{y}\left(p_{\beta}(\cdot-y) \phi(y)\right\}\right.$ has at least one cluster point in $C(G)$ in the topology $U C K$ of bounded and uniform convergence on compact sets. It has precisely one cluster point in $L^{\infty}(\bar{G})$ in the weak* topology since $\left[M_{y}\left(p_{\beta}(\cdot-y) \phi(y)\right)\right]^{\wedge}(\gamma) \equiv p_{\beta}(\gamma) M(\phi \bar{\gamma})$ converges to $M(\phi \bar{\gamma})$ for each $\gamma \in \Gamma$. Denote the $L^{\infty}(\bar{G})$-weak* limit by $\psi$ and refer to a general $U C K$ cluster point by $\omega$. Theorems 9 and 10 to follow concern the ergodic behavior of $\psi$ and $\omega$, respectively. Actually, the second is a corollary of the first, but it also has a very elementary proof, which we give instead.

THEOREM 9. If $\phi$ is ergodic at each $\gamma \neq \gamma_{0} \in \Gamma$ and if $\tau=\psi-\hat{\imath}\left(\gamma_{0}\right) \gamma_{0}$, then the following statements are valid.

(i) For any averaging kernel $\left\{k_{\alpha}\right\}$ on $G$ and for any $p \in A P(G)$ satisfying $M\left(p \bar{\gamma}_{0}\right)=0$, we have

$$
\lim _{\alpha}\left\|\left(k_{\alpha} p\right)^{*} \varphi-p \circ \tau\right\|_{\infty}=0
$$

(ii) Given $\epsilon>0$ there is a neighborhood $U \subset \Gamma$ of $\gamma_{0}$ such that

$$
|\rho(\nu) \circ \tau|_{\infty}<\epsilon\|\nu\|
$$

for every $\nu \in M(G)$ with $\operatorname{supp} \hat{\nu} \subset U$.

Proof. Suppose $\left\{k_{\alpha}\right\}, p$ satisfy the hypothesis of (i). Since $P(G)$ is dense in $A P(G)$, we can assume that $p=\sum a_{i} \gamma_{i} \in P(G)$. In this case

$$
p \circ \tau=\Sigma a_{i} \hat{\tau}\left(\gamma_{i}\right) \gamma_{i}=\Sigma a_{i} M\left(\phi \overline{\gamma_{i}}\right) \gamma_{i}
$$

Since $\gamma_{i} \neq \gamma_{0}$ for each $i$ and since $\phi$ is ergodic at $\gamma \neq \gamma_{0}$, Corollary 3 (iii) yields

$$
\lim _{\alpha}\left\|\left(k_{\alpha} p\right)^{*} \varphi-p \circ \tau\right\|_{\infty}=\lim _{\alpha}\left\|\sum a_{i}\left(k_{\alpha} \gamma_{i}\right)^{*} \varphi-\sum a_{i} M\left(\varphi \bar{\gamma}_{i}\right) \gamma_{i}\right\|_{\infty}
$$




$$
\leq \lim _{\alpha} \sum\left|a_{i}\right|\left\|\left(k_{\alpha} \gamma_{i}\right)^{*} \varphi-M\left(\varphi \bar{\gamma}_{i}\right) \gamma_{i}\right\|_{\infty}=0 .
$$

(ii): By replacing $\phi$ with $\phi \overline{\gamma_{0}}$, we can assume that $\phi$ is ergodic at each $\gamma \neq 0 \in \Gamma$. Let $\left\{k_{\alpha}\right\}$ be a Fejér averaging kernel on $G$ and choose an $L^{1}(\bar{G})$ bounded approximate identity $\left\{p_{\beta}\right\}$ from $P(G)$ which satisfies $\hat{p}_{\beta}(0)=$ $\left|p_{\beta}\right|_{1}$. (Evidently $p_{\beta} \geq 0$ for all $\beta$ ). Choose the number $\epsilon>0$ and the index $\beta_{0}$ arbitrarily. Set $D_{\epsilon}=\{z:|z| \leq \epsilon, z$ complex $\}$. Finally, use Theorem 2 to choose $\alpha_{0}$ so that

$$
M(\phi) \subset \overline{\operatorname{co}}\left(R\left(k_{\alpha_{0}}^{*} \phi\right)\right) \subset M V(\phi)+D_{\epsilon} .
$$

We are going to work with the functions $\left(k_{\alpha} p_{\beta_{0}}\right) *\left(k_{\alpha_{0}} * \phi\right)$, which we rewrite as $\sigma_{\alpha}+\phi_{\alpha}$ where

$$
\begin{gathered}
\sigma_{\alpha}=\left(k_{\alpha}\left(p_{\beta_{0}}-1\right)\right) *\left(k_{\alpha 0} * \phi\right) \quad \text { and } \\
\phi_{\alpha}=k_{\alpha} *\left(k_{\alpha_{0}} * \phi\right) .
\end{gathered}
$$

First, $k_{\alpha} p_{\beta_{0}} \geq 0$ and $\left\{\operatorname{supp} \hat{k}_{\alpha}\right\}$ is a neighborhood base for $0 \in \Gamma$; hence there is an $\alpha_{1} \geq \alpha_{0}$ such that

$$
\left\|k_{\alpha} p_{\beta_{0}}\right\|_{1}=\left(k_{\alpha} p_{\beta_{0}}\right)^{\wedge}(0)=\hat{k}_{\alpha}(0) \hat{p}_{\beta_{0}}(0)=1 \quad \text { for all } \alpha \geq \alpha_{1} \text {. }
$$

In particular

$$
\left(k_{\alpha} p_{\beta_{0}}\right)^{*}\left(k_{\alpha_{0}}^{*} \varphi\right) \subset \overline{\mathrm{co}}\left(\operatorname{Tr}\left(k_{\alpha_{0}}^{*} \varphi\right)\right) \subset \overline{\mathrm{co}}(\operatorname{Tr}(\varphi)) \quad \text { for } \alpha \geq \alpha_{1} .
$$

which, together with the Fundamental Characterization Theorem and our choice of $\alpha_{0}$, yields

$$
\begin{gathered}
M V(\phi) \subset \overline{\operatorname{co}}\left(R\left(\sigma_{\alpha}+\phi_{\alpha}\right)\right) \subset \overline{\operatorname{co}}\left(R\left(k_{\alpha_{0}}{ }^{*} \phi\right)\right) \\
\subset M V(\phi)+D_{\epsilon} \text { for } \alpha \geq \alpha_{1} .
\end{gathered}
$$

Since $\sigma_{\alpha}$ converges uniformly to the trigonometric polynomial $q=\left(p_{\beta_{0}}-\right.$ 1) $\circ \rho\left(k_{\alpha_{0}}\right) \circ \tau$ by part (i) of this theorem, (1) implies the existence of an $\alpha_{2} \geq$ $\alpha_{1}$ such that

$$
\overline{\operatorname{co}}\left(R\left(q+\phi_{\alpha}\right)\right) \subset M V(\phi)+D_{2 \epsilon} \text { for } \alpha \geq \alpha_{2}
$$

Our next step is to prove that $\|q\|_{\infty} \leq 2 \epsilon$. For each $\delta>0$ there is a compact symmetric set $K \subset G$ and an $\alpha_{3} \geq \alpha_{2}$ such that 

(a) $R(q) \subset\{q(k+x) \mid k \in K\}+D_{\delta}$ for any $x \in G$, and
(b) $\left|\phi_{\alpha}(y)-\phi_{\alpha}(x)\right|<\delta$ whenever $x-y \in K, \alpha \geq \alpha_{3}$.

Here (3) (a) is an immediate consequence of almost periodicity of $q$; specifically, $\operatorname{Tr}(q)$ is totally bounded. To get (3) (b) choose $g \in L^{1}(G)$ so that $\hat{g} \equiv 1$ on some neighborhood $U$ of $0 \in \Gamma$ and so that $\left\|g_{x}-g_{y}\right\|_{1}<$ $\delta /\left(\|\phi\|_{\infty}+1\right)$ for all $x, y \in K$ (see the second half of the proof to Theorem 2.6.3 in [11]). Then, for $\alpha_{3}$ sufficiently large, we have $\sigma\left(\phi_{\alpha}\right) \subset U$ whenever $\alpha$ $\geq \alpha_{3}$. Hence

$$
\left\|\left(\phi_{\alpha}\right)_{x}-\left(\phi_{\alpha}\right)_{y}\right\|_{\infty} \leq\left\|g_{x}-g_{y}\right\|_{1}\|\phi\|_{\infty}<\delta
$$

for $\alpha \geq \alpha_{3}$ and for all $x_{2} y \in K$. At any rate, (3) (a) and (b) yield

$$
R(q)+R\left(\phi_{\alpha}\right) \subset R\left(q+\phi_{\alpha}\right)+D_{2 \delta} \text { for } \alpha \geq \alpha_{3},
$$

which together with (2) implies

$$
\overline{\operatorname{co}}(R(q))+\overline{\operatorname{co}}\left(R\left(\phi_{\alpha}\right)\right) \subset M V(\phi)+D_{2 \epsilon}+D_{2 \delta} \text { for } \alpha \geq \alpha_{3} .
$$

Since $M V(\phi) \subset \overline{\operatorname{co}}\left(R\left(\phi_{\alpha}\right)\right)$ by Theorem 2, we conclude that $\overline{\operatorname{co}}(R(q)) \subset D_{2 \epsilon}$ $+D_{2 \delta}$ for all $\delta>0$. Therefore

$$
\|q\|_{\infty} \leq 2 \epsilon
$$

We have just shown that

$$
\left|\left(p_{\beta_{0}}-1\right) \circ \rho\left(k_{\alpha_{0}}\right) \circ \tau\right|_{\infty} \leq 2 \epsilon
$$

for every $\beta_{0}$. Since

$$
\left(p_{\beta_{0}}-1\right) \circ \rho\left(k_{\alpha_{0}}\right) \circ \tau \equiv p_{\beta_{0}} \circ \rho\left(k_{\alpha_{0}}\right) \circ \tau
$$

and since $\left\{p_{\beta}\right\}$ is an $L^{1}(\bar{G})$ bounded approximate identity, this implies that $\left|\rho\left(k_{\alpha_{0}}\right) \circ \tau\right|_{\infty} \leq 2 \epsilon$. According to the Wiener-Lévy Theorem, there exists an $g \in L^{1}(G)$ such that $\left(g^{*} k_{\alpha_{0}}\right)$ " $\equiv 1$ on a neighborhood $U$ of $0 \in \Gamma$ and such that $\|g\|_{1} \leq 1 / \hat{k}_{\alpha_{0}}(0)+\epsilon=1-\epsilon$. Thus $\left|\rho\left(g^{*} k_{\alpha_{0}}\right) \circ \tau\right|_{\infty}<3 \epsilon$ (assume that $\epsilon<1 / 2)$. Now suppose $\nu \in M(G)$ with supp $\hat{\nu} \in U$. Then $\nu^{*} g^{*} k_{\alpha_{0}} \equiv \nu$ and

$$
|\rho(\nu) \circ \tau|_{\infty} \leq\|\nu\|\left|\rho\left(g^{*} k_{\alpha_{0}}\right) \circ \tau\right|_{\infty}<3 \epsilon\|\nu\| .
$$


Recall that, in the paragraph preceding Theorem 9, we defined $\omega$ to be any of the $U C K$ cluster points to the $q_{\beta}(\cdot) \equiv M_{y}\left(p_{\beta}(\cdot-y) \phi(y)\right)$.

THEOREM 10. In the above notation, if $\phi$ is ergodic at $\gamma_{0}$, then $\omega$ is also and $M\left(\omega \bar{\gamma}_{0}\right)=M\left(\phi \bar{\gamma}_{0}\right)$.

Proof. Suppose $\phi$ is ergodic at $\gamma_{0}$ and let $\left\{k_{\alpha}\right\}$ be an averaging kernel on $G$. Since

$$
k_{\alpha}^{*}\left(q_{\beta} \bar{\gamma}_{0}\right)=M_{y}\left(p_{\beta}(\cdot-y)\left(k_{\alpha} * \phi \bar{\gamma}_{0}\right)(y)\right),
$$

we can write

$$
\begin{aligned}
\| k_{\alpha}^{*}\left(q_{\beta} \bar{\gamma}_{0}-\right. & \left.M\left(\varphi \bar{\gamma}_{0}\right)\right)\left\|_{\infty} \leq M\left(\left|p_{\beta}\right|\right)\right\| k_{\alpha}^{*}\left(\varphi \bar{\gamma}_{0}-M\left(\varphi \bar{\gamma}_{0}\right)\right) \|_{\infty} \\
& +\left|M\left(\varphi \bar{\gamma}_{0}\right)\right|\left|\hat{p}_{\beta}(0)-1\right| .
\end{aligned}
$$

Therefore, taking the lim sup of both sides over $\beta$, we have

$$
\left\|k_{\alpha}{ }^{*}\left(\omega \bar{\gamma}_{0}-M\left(\varphi \bar{\gamma}_{0}\right)\right)\right\|_{\infty} \leq \| k_{\alpha}^{*}\left(\varphi \bar{\gamma}_{0}-M\left(\varphi \bar{\gamma}_{0}\right) \|_{\infty} \lim \sup M\left(\left|p_{\beta}\right|\right),\right.
$$

for all $\alpha$. Now Corollary 3 implies that $\omega$ is ergodic at $\gamma_{0}$ and that $M\left(\omega \bar{\gamma}_{0}\right)=$ $M\left(\phi \overline{\gamma_{0}}\right)$.

We note that the $\omega$ of Theorem 10 need not be ergodic at $\gamma_{0}$ if $\phi$ is not ergodic at $\gamma_{0}$.

Ergodic sets. The main result of this section is Theorem 12 which concerns the union of an ergodic set $E$ with a compact scattered set $S$. When $E$ is Helson, Corollary 13 provides an interesting decomposition for the $\phi \in \Phi(E \cup S)$. These results depend heavily on the previous sections. We conclude with a very elementary proof of a theorem due to Rosenthal and Veech.

For a discussion of ergodic sets, see the introduction. Here we restrict the preliminaries to the formal definition. Recall that $\Phi(E)$ is the closure of span $E$ in $C(G)$ under the relative $L^{\infty}(G)$-weak* topology.

Definition 11. A compact subset $E \subset \Gamma$ is ergodic if each $\phi \in \Phi(E)$ is ergodic.

THEOREM 12. The union of an ergodic set $E$ and a compact scattered set $S$ is ergodic. 
Proof. Arbitrarily choose $\phi \in \Phi(E \cup S)$. We first point out that since $S$ is closed and scattered, each $\psi \in \Phi(E \cup S)$ with $\sigma(\psi) \subset E$ is actually an element of $\Phi(E)$ (note that $E$ is a spectral set if and only if $\sigma(\psi) \subset E \Longleftrightarrow \psi \in$ $\Phi(E))$. Together with the "invariance" of ergodicity at $\gamma$ under convolution, this means that $\phi$ is ergodic at each $\gamma \in E \cap S^{c}$. Of course, $\phi$ is ergodic on $(E \cup S)^{c} \subset \sigma(\phi)^{c}$. Thus if $Q$ consists of all points at which $\phi$ is not ergodic, then $Q \subset S$. If $Q=\phi$, then $\phi$ is ergodic and we are done. Thus suppose $Q$ $\neq \varnothing$.

Since $Q$ is scattered, there is a point $\gamma_{0} \in Q$ and a $g \in L^{1}(G)$ such that $\hat{g}\left(\gamma_{0}\right)=1$ and supp $\hat{g} \cap Q=\left\{\gamma_{0}\right\}$. The function $g * \phi$ is ergodic where $\phi$ is ergodic and is also ergodic on $\sigma\left(g^{*} \phi\right)^{c}$. Hence $g^{*} \phi$ is ergodic on $\left\{\gamma_{0}\right\}^{c}$. Furthermore $g^{*} \phi$ is not ergodic at $\gamma_{0}$ since $M\left[\left(g^{*} \phi\right) \bar{\gamma}_{0}\right]=\bar{g}\left(\gamma_{0}\right) M\left(\phi \overline{\gamma_{0}}\right)=$ $M\left(\phi \overline{\gamma_{0}}\right)$ for every invariant mean $M$ on $C(G)$. Fix the mean $M$ and choose an $L^{1}(G)$ bounded approximate identity $\left\{p_{\beta}\right\} \subset P(G)$. Define

$$
\begin{aligned}
q_{\beta} & =M_{y}\left[\left(p_{\beta}(\cdot-y)-\hat{p}_{\beta}\left(\gamma_{0}\right) \gamma_{0}(\cdot-y)\right) g^{*} \phi(y)\right] \\
& =M_{y}\left(p_{\beta}(\cdot-y) g * \phi(y)\right)-\hat{p}_{\beta}\left(\gamma_{0}\right) M\left(\phi \bar{\gamma}_{0}\right) \gamma_{0} .
\end{aligned}
$$

Denote the $L^{\infty}(\bar{G})$-weak* limit of $\left\{q_{\beta}\right\}$ by $\tau$ and observe that $g^{*} \phi, \tau$ replaces the $\phi, \tau$ of Theorem 9 , respectively. Furthermore, $p_{\beta}{ }^{\circ} \tau=q_{\beta}$ for all $\beta$. Let $\omega \in C(G)$ be one of the cluster points to $\left\{q_{\beta}\right\}$ in the topology of uniform convergence on compact subsets of $G$. Choose a Fejerr averaging kernel $\left\{k_{\alpha}\right\}$ on $G$ and an upper bound $K$ for $\left\{\left|p_{\beta}\right|_{1}\right\}$. Since $\left\{\operatorname{supp}\left(k_{\alpha} \gamma_{0}\right)\right\}$ is a neighborhood base for $\gamma_{0}$, Theorem 9 (ii) and the convergence of $q_{\beta}$ to $\omega$ yield

$$
\begin{aligned}
\left\|\left(k_{\alpha} \gamma_{0}\right)^{*} \omega\right\|_{\infty} & \leq \lim _{\beta} \sup \left\|\left(k_{\alpha} \gamma_{0}\right)^{*} q_{\beta}\right\|_{\infty}=\lim _{\beta} \sup \left\|\left(k_{\alpha} \gamma_{0}\right)^{*}\left(p_{\beta} \circ \tau\right)\right\|_{\infty} \\
& \leq K \dot{I} \rho\left(k_{\alpha} \gamma_{0}\right) \circ \tau \coprod_{\infty}<K \epsilon\left\|k_{\alpha} \gamma_{0}\right\|_{1}=\epsilon K,
\end{aligned}
$$

for all $\alpha$ sufficiently large, for each $\epsilon>0$ (sufficiently large depends on $\epsilon$ ). Corollary 3 (iii) now implies that $\omega$ is ergodic at $\gamma_{0}$ and that $M\left(\omega \bar{\gamma}_{0}\right)=0$. On the other hand, for $\gamma \neq \gamma_{0}$ Theorem 10 tells us that $\omega$ is ergodic at $\gamma$ and that $M\left[\left(g^{*} \phi\right) \bar{\gamma}\right]=M(\omega \bar{\gamma})$. In particular, $g^{*} \phi-\omega$ is ergodic on $\left\{\gamma_{0}\right\}^{c} ; L\left[\left(g^{*} \phi\right.\right.$ $-\omega) \bar{\gamma}]=0$ for each $\gamma \neq \gamma_{0}$; and $L\left[\left(g^{*} \phi-\omega\right) \bar{\gamma}_{0}\right]=L\left[\left(g^{*} \phi\right) \bar{\gamma}_{0}\right]$. Here $L$ is any invariant mean on $C(G)$.

We are now in a position to show that $\sigma\left(g^{*} \phi-\omega\right) \subset E$. To this end, arbitrarily choose $h \in L^{1}(G)$ with supp $\hat{h} \cap E=\varnothing$. Then $\sigma\left(h^{*}\left(g^{*} \phi-\right.\right.$ $\omega)) \subset E^{c} \cap(E \cup S)=E^{c} \cap S$, which is a scattered set. By an elementary argument [5, Theorem 2.4], either $h^{*}\left(g^{*} \phi-\omega\right) \equiv 0$ or it is ergodic at least 
at some isolated $\xi \in E^{c} \cap S$ with $M\left[h^{*}\left(g^{*} \phi-\omega\right) \xi\right] \neq 0$. Suppose the latter is true. Since $M\left[h^{*}\left(g^{*} \phi-\omega\right) \xi\right]=\hat{h}(\xi) M\left[\left(g^{*} \phi-\omega\right) \xi\right] \neq 0$, we conclude that $\xi=\gamma_{0}$ and that $g^{*} \phi-\omega$ is ergodic at $\gamma_{0}$. But then $g^{*} \phi$ is ergodic at $\gamma_{0}$, which is false. Thus $h^{*}\left(g^{*} \phi-\omega\right) \equiv 0$, and we conclude that $\sigma\left(g^{*} \phi-\omega\right) \subset E$.

Finally, $g^{*} \phi-\omega \in \Phi(E \cup S)$ and $\sigma(g * \phi-\omega) \subset E$ imply $g * \phi-\omega$ $\in \Phi(E)$. But $E$ is ergodic. Thus $g^{*} \phi-\omega$ is certainly ergodic at $\gamma_{0}$. Since $\omega$ is also, we conclude that $g^{*} \phi$ is ergodic at $\gamma_{0}$, which is again false. The remaining possibility is $Q=\varnothing$.

We want to point out that Theorems 8 and 12 imply that any $\phi \in C(G)$ with scattered spectrum is in the uniform closure of $P(G)$ and is therefore almost periodic. This result, which is due to Loomis [9], cannot be used to simplify the proof of Theorem 12 in a significant manner. The difficulty arises from the fact that there are functions which are nonergodic at precisely one point in $\Gamma$. To deal with this difficulty in the setting of Theorem 12 one needs Theorem 9 (ii) or a similar result. Another extension of the Loomis result is the following corollary which concerns Helson sets. (See [11, Section 5.6] for a discussion of Helson sets). Denote by $M_{c}(E)$ the set of continuous $\mu \in M(\Gamma)$ with measure support in $E$. Recall that $\hat{\mu}(x)=$ $\int \Gamma(\gamma, x) d \mu(\gamma)$, for $\mu \in M(\Gamma)$.

Corollary 13. Suppose E is Helson and $S$ is compact scattered. Then each $\phi \in \Phi(E \cup S)$ decomposes uniquely into

$$
\phi=\hat{\mu}+p \text {, where } \mu \in M_{c}(E) \text { and } p \in A P(G) \text {. }
$$

Proof. According to Theorem 12, $\phi$ is ergodic. Hence Theorem 8 asserts the existence of a $(\phi, S)$-pair $\left\{g_{n}\right\},\left\{p_{n}\right\}$. Since $\sigma\left(g_{n} * \phi\right) \subset E$ and $g_{n}{ }^{*} \phi \in \Phi(E \cup S)$, we have $g_{n}{ }^{*} \phi \in \Phi(E)$. But $E$ is Helson; thus $g_{n}{ }^{*} \phi=$ $\hat{\nu_{n}}$ for some $\nu_{n} \in M(E)$. In particular $\left\{g_{n} * \phi+p_{n}\right\}$ is a sequence of weakly almost periodic functions which converges uniformly to $\phi$. According to [3, Theorem12.1], $\phi$ must therefore be weakly almost periodic. Thus $\phi$ decomposes into $\phi=w+p$ where $p \in A P(G), \sigma(p) \subset \sigma(\phi)$, and $M(|w|)=0$ for all invariant means $M$ on $C(G)[4$, Theorem 1]. Using an argument presented in the proof of Theorem 12, we conclude that $\sigma(w) \subset E$. Thus $w$ $\in \Phi(E)$. Since $E$ is Helson, $w=\hat{\mu}$ for some $\mu \in M(E)$. Since $M(|w|)=0, \mu$ $\in M_{c}(E)$. The uniqueness of this decomposition is immediate.

This corollary suggests the possibility of decomposing the general ergodic function in a manner somewhat analogous to the decomposition of weakly almost periodic functions [4]. It turns out that no reasonable 
decomposition is possible. For a complete discussion of this problem see [14].

Our last theorem is due to Rosenthal and Veech [12]. We include it here since our proof depends only on the elementary properties of invariant means and the Loomis result.

THEOREM. Let $E \subset \Gamma$ be a set of spectral synthesis. If $S \subset \Gamma$, is scattered and if $E \cup S$ is closed, then $E \cup S$ is a set of spectral synthesis.

Proof. Assume that $E, S$ satisfy the hypothesis. Note that if $V \subset E^{c}$ is a compact set, then $V \cap S$ is a compact scattered set.

We must show that each $\phi \in C(G)$ with compact spectrum contained in $E \cup S$ is in $\Phi(E \cup S)$. To this end suppose $\phi \in C(G), \sigma(\phi) \subset E \cup S$, and $\sigma(\phi)$ is compact. Let $M$ be a fixed invariant mean on $C(G)$ and let $\left\{p_{\alpha}\right\} \subset$ $P(G)$ be an $L^{1}(\bar{G})$ bounded approximate identity. Set $q_{\alpha}=$ $M_{y}\left(p_{\alpha}(\cdot-y) \phi(y)\right)$. Observe that $q_{\alpha} \in \Phi(E \cup S)$ and that $\left\|q_{\alpha}\right\|_{\infty}$ is uniformly bounded in $\alpha$. According to the remarks preceding Theorem 9, there is a subnet $\left\{q_{\beta}\right\}$ of $\left\{q_{\alpha}\right\}$ which converges in the relative $L^{\infty}(G)$-weak* topology to an $\omega \in C(G)$. The Loomis result [9], together with Theorem 10, implies that $L[(\phi-\omega) \bar{\gamma}]=0$ for all $\gamma \in E^{c}$ and for all invariant means $L$. It follows that $\sigma(\phi-\omega) \subset E \cup S$ has no isolated points in $E^{c}$. Hence $\sigma(\phi-\omega)$ $\subset E$, a set of spectral synthesis. Therefore $\phi \equiv(\phi-\omega)+\omega \in \Phi(E \cup S)$.

\section{REFERENCES}

1. A. S. Besicovitch, Almost Periodic Functions, Dover Publications, Inc., New York, 1964. 2. R. B. Burckel, Weakly Almost Periodic Functions on Semigroups, Gordon and Breach Science Publishers, New York, 1970.

3. W. F. Eberlein, Abstract ergodic theorems and weak almost periodic functions, Trans. Am. Math. Soc., 67 (1949), 217-240.

4. $\longrightarrow$ The point spectrum of weakly almost periodic functions, Michigan J., 3 (1955-1956), 137-139.

5. C. S. Herz, The spectral theory for bounded functions, Trans. Amer. Math. Soc., 94 (1960), 181-232.

6. J. -P. Kahane, Sur les fonctions presque-périodiques généralisées sont le spectre est vide, Studia Math. 21 (1962), 231-236.

7. Y. Katznelson and O. MeGehee, Measures and pseudomeasures on compact subsets of the line, Math. Scand.,23 (1968), 57-68.

8. B. M. Levitan, Some questions of the theory of almost periodic functions, Amer. Math. Soc. Transl., 28 (1950), 53 pp.

9. L. H. Loomis, The spectral characterization of a class of almost periodic functions, Ann. Math., 72 (1960), 362-368.

10. G. G. Lorentz, A contribution to the theory of divergent sequences, Acta Math., 80 (1948), 167-190.

11. W. Rudin, Fourier Analysis on Groups, Interscience Tracts in Pure and Applied 
Mathematics, Interscience Publishers, Inc., New York, 1967.

12. W. A. Veech, Properties of minimal functions on abelian groups, Amer. J. Math., 91 (1969), 415-440.

13. G. S. Woodward, Sur une classe d'ensembles épars, C. R. Acad. Sci. Paris., 274 (1972), 221-223.

14. $\longrightarrow$ The generalized almost periodic part of an ergodic function, Studia Math., 50 (1974), 103-116.

Received January 3, 1973 and in revised form August 12, 1974. This paper is part of the author's dissertation which was written under the direction of Professor C. Robert Warner at the University of Maryland and completed in August 1971.

UNIVERSITY OF NEBRASKA 



\section{PACIFIC JOURNAL OF MATHEMATICS}

\section{EDITORS}

RICHARD ARENS (Managing Editor)

University of California

Los Angeles, California, 90024

\section{R. A. BEAUMONT}

University of Washington

Seattle, Washington 98105

\section{J. DugunDJI}

Department of Mathematics University of Southern California Los Angeles, California 90007

D. Gillbarg AND J. MilgRAM

Stanford University Stanford, California 94305

\section{ASSOCIATE EDITORS}

E. F. BECKENBACH

B. H. NEUMANN

SUPPORTING

UNIVERSITY OF BRITISH COLUMBIA

UNIVERSITY OF CALIFORNIA

MONTANA STATE UNIVERSITY

UNIVERSITY OF NEVADA

NEW MEXICO STATE UNIVERSITY

OREGON STATE UNIVERSITY

UNIVERSITY OF OREGON OSAKA UNIVERSITY
F. WOLF

K. YosHIDA

INSTITUTIONS

UNIVERSITY OF SOUTHERN CALIFORNIA

STANFORD UNIVERSITY

UNIVERSITY OF TOKYO

UNIVERSITY OF UTAH

WASHINGTON STATE UNIVERSITY

UNIVERSITY OF WASHINGTON

AMERICAN MATHEMATICAL SOCIETY

NAVAL WEAPONS CENTER

The Supporting Institutions listed above contribute to the cost of publication of this Journal, but they are not owners or publishers and have no responsibility for its content or policies.

Mathematical papers intended for publication in the Pacific Journal of Mathematics should be in typed form or offset-reproduced, (not dittoed), double spaced with large margins. Underline Greek letters in red, German in green, and script in blue. The first paragraph or two must be capable of being used separately as a synopsis of the entire paper. Items of the bibliography should not be cited there unless absolutely necessary, in which case they must be identified by author and Journal, rather than by item number. Manuscripts, in duplicate if possible, may be sent to any one of the five editors. Please classify according to the scheme of Math. Rev. Index to Vol. ${ }^{39}$. All other communications to the editors should be addressed to the managing editor, or Elaine Barth, University of California, Los Angeles, California, 90024.

100 reprints are provided free for each article, only if page charges have been substantially paid. Additional copies may be obtained at cost in multiples of 50 .

The Pacific Journal of Mathematics is issued monthly as of January 1966. Regular subscription rate: $\$ 72.00$ a year (6 Vols., 12 issues). Special rate: $\$ 36.00$ a year to individual members of supporting institutions.

Subscriptions, orders for back numbers, and changes of address should be sent to Pacific Journal of Mathematics, 103 Highland Boulevard, Berkeley, California 90708.

\section{PUBLISHED BY PACIFIC JOURNAL OF MATHEMATICS, A NON-PROFIT CORPORATION}

Copyright (C) 1974 by Pacific Journal of Mathematics

Manufactured and first issued in the U.S.A. 


\section{Pacific Journal of Mathematics}

\section{Vol. 54, No. $2 \quad$ June, 1974}

John Edward Coury, Walsh series with coefficients tending monotonically to

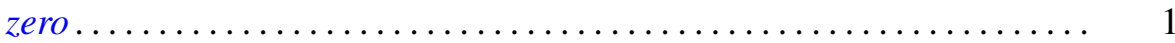

Patrick Michael Fitzpatrick and Walter Volodymyr Petryshyn, Fixed point theorems for multivalued noncompact acyclic mappings ............

Irving Leonard Glicksberg, More on Phragmén-Lindelöf for function

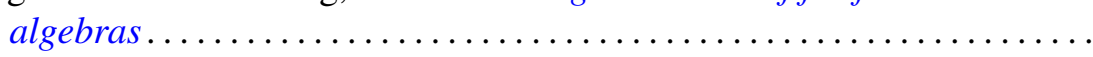

Adilson Goncalves, Structural constants. II .................. 39

Richard P. Gosselin, Closure theorems for affine transformation groups .... 53

Ralph Peter Grimaldi, Baer and UT-modules over domains ........... 59

Edward Grossman, On the prime ideal divisors of $\left(a^{n}-b^{n}\right) \ldots \ldots \ldots \ldots . \ldots 73$

A. Hedayat and Ester Seiden, On the theory and application of sum composition of Latin squares and orthogonal Latin squares.......... .

Gerald L. Itzkowitz, Continuous measures, Baire category, and uniform continuity in topological groups ......................... 115

Francis Masat, Right simple congruences on a semigroup ............ 127

Robert Harvey Oehmke, Right congruences and semisimplicity for Rees matrix semigroups..................................

Qazi Ibadur Rahman and Jan Stankiewicz, Differential inequalities and local valency . . . . . . . . . . . . . . . . . . . . . . . . . . . . . . . . . . . . . 165

William John Reed, Random points in a simplex ................ 183

Mohan S. Shrikhande, Strongly regular graphs and group divisible

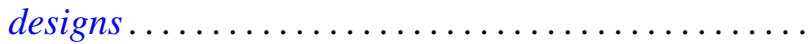

Zahava Shmuely, The structure of Galois connections ... . .

Robert C. Shock, Dual generalizations of the Artinian and Noetherian

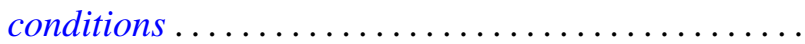

Arne Stray, Approximation and interpolation for some spaces of analytic functions in the unit disc ........................

Eldon Jon Vought, Monotone decompositions into trees of Hausdorff continua irreducible about a finite subset ............

James Wirth, The mapping cylinder axiom for WCHP fibrations ... 263

Gordon S. Woodward, Invariant means and ergodic sets in Fourier analysis... 